\title{
Quasiparticle Diffusion in CRESST Light Detectors
}

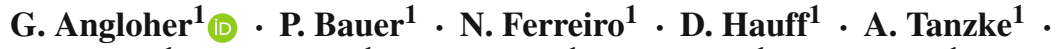 \\ R. Strauss ${ }^{1}$ - M. Kiefer ${ }^{1}$ - F. Petricia ${ }^{1}$ - F. Reindl ${ }^{1}$ - W. Seidel ${ }^{1}$. \\ F. Pröbst ${ }^{1}$ - M. Wüstrich ${ }^{1}$
}

Received: 30 September 2015 / Accepted: 16 January 2016 / Published online: 4 February 2016 (C) The Author(s) 2016. This article is published with open access at Springerlink.com

\begin{abstract}
CRESST-II is a direct dark matter experiment that uses scintillating calorimeters to detect WIMP-induced nuclear scatter processes. Heat and light signals are read out with tungsten transition edge sensors (TESs) that are optimized toward their sensitivity to non-thermal phonons. The usage of superconducting thin film structures (e.g., aluminum) serving as phonon collectors to increase the collection area for this signal component is an approach to improve the sensitivity of the TES. The performance of the phonon collectors depends on the material properties and the quality achieved in the production process. We optimized the size of the phonon collectors for the given quality of CRESST-II light detectors. The diffusion lengths measured in this work are $\mathcal{O}(1 \mathrm{~mm})$ and show a strong correlation to the Residual Resistivity Ratio of the respective films. First tests of CRESST-II light detectors with larger as well as thicker phonon collectors individually show improvements in the measured pulse height of $30 \%$.
\end{abstract}

Keywords Low temperature detector - Quasiparticle diffusion · Transition edge sensor · Dark matter searches · Phonon collectors

\section{Introduction}

CRESST-II is a direct dark matter search that uses scintillating calorimeters measuring heat and scintillation light to detect nuclear recoils in $\mathrm{CaWO}_{4}$ single crystals $[1,2]$. The ratio of the heat signal from the main absorber and the scintillation light signal is used to discriminate between $\gamma / \beta$-events, $\alpha$-events and nuclear recoils (e.g., neutrons) and

M. Wüstrich

marc.wuestrich@mpp.mpg.de

1 Max-Planck-Institute f. Physics, Föhringer Ring 6, 80805 Munich, Germany 

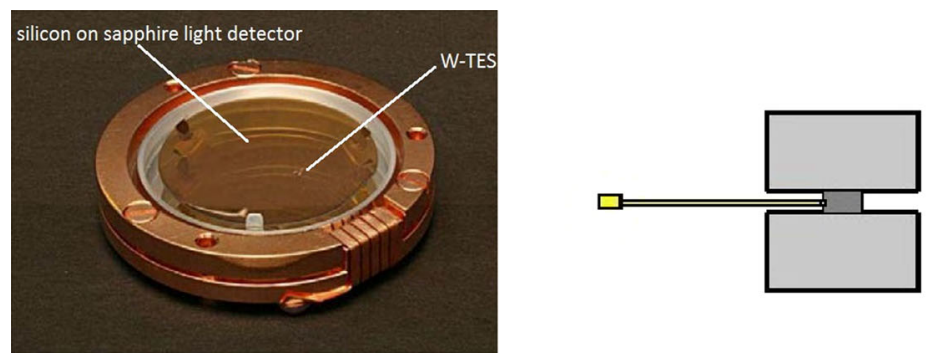

미

Fig. 1 Left Standard SOS light detector with $40 \mathrm{~mm} \varnothing$ mounted in a CRESST-II light detector holder. Right Standard TES structure used in CRESST-II. The tungsten part (dark gray) is largely covered by aluminum (light gray). The weak thermal link is provided via a gold strip. An external, electrically decoupled heater which is also evaporated onto the SOS substrate is used to stabilize the TES in its operation point (Color figure online)

enables CRESST-II to reject background efficiently. The main absorber is equipped with a tungsten Transition Edge Sensor (TES) with transition temperature $T_{\mathrm{C}}=15 \mathrm{mK}$ measuring the temperature rise of the $\mathrm{CaWO}_{4}$ crystal due to particle interactions. The scintillation light is detected with a spatially separated silicon-on-sapphire (SOS) light detector, which is also equipped with tungsten TES. The standard CRESST-II light detector consists of a $40 \mathrm{~mm}$ diameter SOS wafer with a mass of $21 \mathrm{~g}$ (see Fig. 1 left). The baseline noise in the current physics run of CRESST-II is $\approx 5 \mathrm{eV}$, which nearly allows to detect single photons. The tungsten TES (see Fig. 1) (dark gray) is largely covered with superconducting aluminum phonon collectors (light gray) to increase its sensitivity to non-thermal phonons. A gold strip, coupling the TES weakly to the thermal bath, serves as the thermal link. An external, electrically decoupled heater which is also evaporated onto the SOS substrate is used to stabilize the TES in its operation point.

Phonon collectors are an elegant approach to overcome the dilemma of increasing the collection area $A_{\text {TES }}$ for the non-thermal phonons, while keeping the overall heat capacity of the TES small $\left(\propto \mathrm{m}_{\mathrm{W}}\right)[3,4]$. In a phonon collector, Cooper Pairs are split into excited quasiparticles (free $e^{-}$), which loose energy, while diffusing due to scattering on crystal defects and surfaces and finally recombine to Cooper Pairs. Excited quasiparticles decay emitting phonons which break more Cooper Pairs to quasiparticles as long their energy $E_{\mathrm{QP}}$ exceeds twice the band gap $E_{\mathrm{gap}}$ [4]. Since the recombination to Cooper Pairs can lead to signal losses, the transport of the quasiparticles to the tungsten TES and the collection of the quasiparticles therein is crucial to improve the signal height. Therefore, it is necessary to measure the diffusion length of the quasiparticles in the superconducting material to be able to dimension the phonon collectors accordingly. The diffusion length depends strongly on the materials used as phonon collectors and on the quality achieved in their production.

\section{Setup}

To measure the diffusion length for CRESST-II light detectors, a dedicated setup is used (see Fig. 2). A silicon substrate is equipped with two tungsten TESs that are connected 


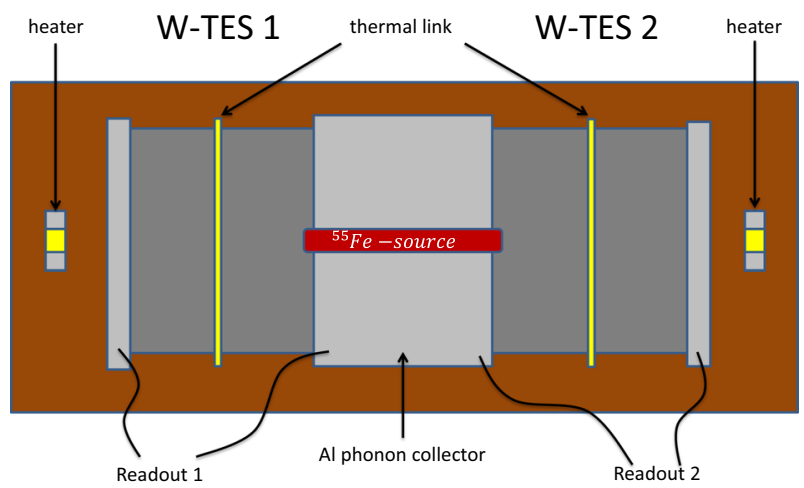

Fig. 2 Experimental setup for the measurement of the diffusion length in CRESST light detectors. A silicon carrier is equipped with two tungsten TESs (dark gray) that are connected with a single superconducting aluminum phonon collector (light gray). Equipped with two heaters, the TESs can be also operated if their transition temperature $T_{\mathrm{C}}$ is different. Illuminated by an X-ray source (red area), events being created in the phonon collector can be read out in a correlated way with two individual readouts (Color figure online)

with a single superconducting aluminum phonon collector. Due to the low thermal conductance of the phonon collector, the two TESs can be operated individually, even if their transition temperature $T_{c}$ is different. A strong thermal link of each TES guarantees a fast relaxation after a particle interaction and allows a high event rate. To allow the stabilization in the correct operation point, each TES is equipped with an electrically separated heater. The phonon collector is illuminated with a X-ray source (e.g., ${ }^{55} \mathrm{Fe}$-source) through a slit with a width of $0.1 \mathrm{~mm}$ and a length of $5 \mathrm{~mm}$. Using only a slim slit for the illumination, the diffusion of $n$ created quasiparticles at a position $x_{\mathrm{A}}$ can be reduced to a problem that can be modeled over the time $t$ by a 1D diffusion equation (Eq. 1) assuming the diffusion length is not mainly limited by the thin film thickness.

$$
\frac{\delta n}{\delta t}-D \cdot \frac{\delta^{2} n}{\delta x^{2}}+\frac{n}{\tau_{\mathrm{qp}}}=0
$$

Therein, the diffusion constant $D=\sqrt{v_{\mathrm{g}} \cdot l / 3}$, the group velocity $v_{\mathrm{g}}$, and the lifetime of the quasiparticles $\tau_{\mathrm{qp}}$ are used. The boundary conditions allow an analytic solution of Eq. 1 in a setup with a distance $L$ between the two TES: $n(t=0, x= \pm L / 2)=0$, $n\left(t=0, x_{a}\right)=N_{0} \cdot \delta\left(t_{0}-t\right)$. The ratio of the number of detected quasiparticles $n_{1,2}$ in TES 1/TES 2 to the number of generated particles $N_{0}(E)$ can be described by Eq. 2 .

$$
\frac{n_{1,2}\left(x_{a}, \alpha, \beta, L\right)}{N_{0}(E)}=\frac{\sinh \left(\alpha\left(0.5 \pm \frac{x_{a}}{L}\right)\right)+\beta \cosh \left(\alpha\left(0.5 \pm \frac{x_{a}}{L}\right)\right)}{\left(1+\beta^{2}\right) \sinh (\alpha)+2 \beta \cos h(\alpha)} .
$$

The parameter $\alpha=L / \sqrt{D \cdot \tau_{\mathrm{qp}}}$ describes the quasiparticle diffusion properties of a phonon collector with the dimension $L . \beta=\tau_{\text {tr }} / \tau_{\mathrm{qp}}$ takes into account that the trapping time $\tau_{\text {tr }}$ of the quasiparticles in the area of the tungsten TES ( $\geq$ reduced band gap) is not zero and diffusion back into the phonon collector is possible. 

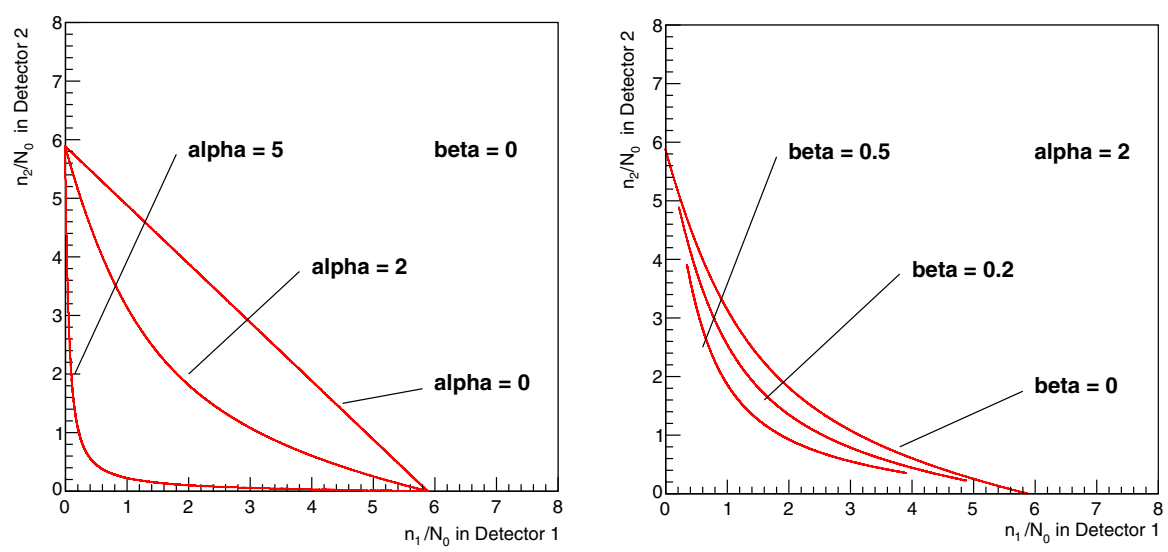

Fig. 3 Simulation of mono-energetic events randomly distributed through the slit of the setup for different values of $\alpha$ (left) and $\beta$ (right). The shape of the event distribution strongly depends on the two diffusion parameters and allows the extraction of each parameter with high precision (Color figure online)

Therefore, $\beta$ includes the finite collection efficiency of the TES. To test the influence if the individual parameters on the diffusion, a dedicated simulation was developed. When the setup is illuminated with a mono-energetic X-ray source, a signal distribution is expected as depicted in Fig. 3. Due to the loss while diffusing the sum of the signals is not constant but form for mono-energetic events a scatter distribution that resembles a "banana" $(\alpha>0)$. The shape of the scatter distribution of mono-energetic events strongly depends on the parameters $\alpha$ and $\beta$ and allows to extract the diffusion length by fitting the "banana" with $n_{2}\left(n_{1}, \alpha, \beta, x_{\mathrm{A}}\right) / N_{0}$. Systematic errors introduced by the fit are negligible compared to the systematic errors introduced by the experimental setup, mainly by the determination of the absorption point $x_{\mathrm{A}}$ (see Sect. 3).

\section{Analysis and Results}

To investigate the diffusion parameter of CRESST-II light detectors, four independent measurements with the same TES layout were performed. The carrier is a $20 \times 10 \times 0.4$ mm silicon substrate, which is equipped with an detector layout as depicted in Fig. 2. The distance $L$ between the two TESs is $4 \mathrm{~mm}$. For the illumination with X-rays, an ${ }^{55} \mathrm{Fe}$-source is used. Figure 4 shows the recorded scatter plot with the recorded pulse height of the respective TESs on the two axes. "banana"-shaped scatter distributions show a wide range of the TES pulse heights. Four main contributions can be assigned: the phonon collector hits and substrate hits created by the $K_{\alpha}$ and $K_{\beta}$ lines of the ${ }^{55} \mathrm{Fe}$-source. The ratio of the two distributions is in agreement with the absorption probability of these x-ray energies in the respective layers. In the very outer regions of the phonon collector, the shape of the "banana" is influenced by the strong thermal link of the TESs and the pulse heights decrease. For the determination of the diffusion length, these events are not considered. Furthermore, for the determination of the diffusion length, only events absorbed in the phonon collector itself are considered. 


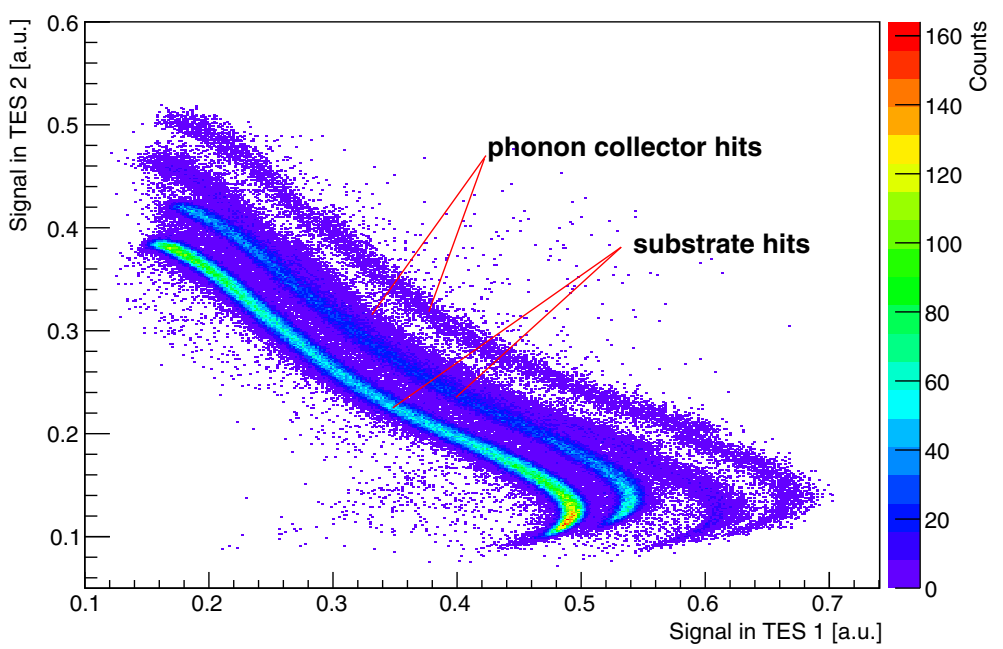

Fig. 4 Scatter plot of recorded data in measurement 1. The axes show the respective pulse height measured in the two TESs for a particle interaction. Over a broad distribution of pulse heights, the "banana" is well pronounced. Four scatter distributions can be attributed to hits created by the $K_{\alpha}$ and $K_{\beta}$ lines of the ${ }^{55} \mathrm{Fe}-$ source being absorbed in the phonon collector or in the substrate underneath. On the very edges, where the events correspond to hits very close to one of the TESs and to their strong thermal link, the "banana" stops and the pulse heights decrease rapidly. Since these data points have different physical properties concerning their diffusion, they are removed from the diffusion analysis and only the "banana" itself is considered (Color figure online)

To fix the position the event took place, the scatter plot is divided in cake slices having their origin at zero. By doing this, it is possible to precisely extract the mono-energetic events of the ${ }^{55} \mathrm{Fe} \mathrm{X}$-ray spectrum at a certain absorption point $x_{\mathrm{A}}$. Due to the limited amount of events, the slices have a finite width and, therefore, introduce a systematic error which is dominating all other uncertainties. The peak positions extracted are used to determine the exact shape of the banana which is ultimately fitted to extract the diffusion length (see Fig. 3). The results of all measurements performed are listed in Table 1. The diffusion parameters obtained with the measurements are in the order of $\mathcal{O}(1 \mathrm{~mm})$ and exceed previous expectations. Though, large fluctuations can be observed between the different measurements. Those can be mainly explained by a different quality of the setups, since the quasiparticle diffusion depends on the quality of the phonon collector. This is in good agreement with Residual Resistivity Ratio (RRR) measurements performed for all films, showing that the number of crystal defects has a impact which can be correlated on the diffusion length. Concerning the influence of the large values of $\alpha$ on the fit uncertainty, one can notice that only a small fraction of the "banana" is recorded causing a lower a sensitivity of the fit to its parameters. Still, the uncertainty originating from the determination of the absorption point $x_{\mathrm{A}}$ is dominating the combined uncertainty.

The standard geometry of the CRESST-II light detectors allows a diffusion of quasiparticles of $\approx 0.2 \mathrm{~mm}$. With a diffusion length in the order of $\mathcal{O}(1 \mathrm{~mm})$, the dimensions of the phonon collectors can be increased. To investigate these changes, light detector substrates are equipped with a standard TES structure as well as with a TES with an increased phonon collector area and tested simultaneously. This method 
Table 1 Summary of the results

\begin{tabular}{lclc}
\hline & \multicolumn{1}{l}{$\alpha$} & Diffusion length $(\mathrm{mm})$ & RRR \\
\hline Measurement 1 & $1.48 \pm 0.02$ & $2.94 \pm 0.23$ & 96 \\
Measurement 2 & $10.08 \pm 0.07$ & $0.56 \pm 0.09$ & 54 \\
Measurement 3 & $8.98 \pm 0.07$ & $0.63 \pm 0.16$ & 62 \\
Measurement 4 & $2.33 \pm 0.3$ & $1.21 \pm 0.21$ & 82 \\
\hline
\end{tabular}

The errors are a combination of the statistical errors of the fits and the systematic uncertainty given by the setup (dominated by extraction of $x_{A}$ ). Since $\beta$ is measured to be very small in all measurements, it is not listed. The results vary in the order $\mathcal{O}(1 \mathrm{~mm})$ and show a large correlation to the RRR of the phonon collectors

minimizes systematic errors that are linked to the production process and promises a better comparability. With this method, we found firstly that by doubling the phonon collector area, the signal height can be increased by $30 \%$ compared to a standard geometry. Likewise, increasing the thickness of the phonon collectors by a factor of 5 while keeping the phonon collector area constant improves the signal by $30 \%$ and shows that in our case the diffusion length is not only dominated by crystal defects in the phonon collector but also by scattering on its surfaces. Therefore, the model describing the detector has to be adapted to consider the influence of the thickness on the fit parameters.

\section{Summary}

The quality of the phonon collectors used for CRESST-II light detectors (CRESST-II (Phase 2)) was underestimated. The measurements performed for this work show that the diffusion length of quasiparticles generated in the phonon collectors is $\mathcal{O}(1 \mathrm{~mm})$. The fluctuations of the values can be explained with the correlation of the diffusion length on the film quality of the phonon collectors (see RRR measurements). Based on the obtained information, the TES of our light detectors is adapted to the longer diffusion lengths, particularly since measurements with increased phonon collector dimensions (area and thickness) show an significant improvement of the detector performance. Latest results point to the fact that the diffusion length of quasiparticles in CRESST-II light detectors is not limited to the quality of the phonon collectors but by mainly by the thickness of the aluminum (further investigations necessary). The findings of this work are implemented in the future CRESST-III runs and promise, in combination with a reduced light detector mass, a resolution that allows single-photon detection $(<3 \mathrm{eV})$. In of the light dark matter scenario and the quest of very low detector thresholds, the achieved improvement of CRESST light detectors is crucial to guarantee particle discrimination even at the lowest recoil energies.

Acknowledgments Open access funding provided by Max-Planck-Institute for Physics.

Open Access This article is distributed under the terms of the Creative Commons Attribution 4.0 International License (http://creativecommons.org/licenses/by/4.0/), which permits unrestricted use, distribution, 
and reproduction in any medium, provided you give appropriate credit to the original author(s) and the source, provide a link to the Creative Commons license, and indicate if changes were made.

\section{References}

1. G. Bertone, D. Hooper, J. Silk, Phys. Rep. 405, 279-390 (2005). doi:10.1016/j.physrep.2004.08.031

2. G. Angloher et al., CRESST Collaboration. Eur. Phys. J. C 74, 3184 (2014). doi:10.1140/epjc/ s10052-014-3184-9

3. F. Pröbst et al., J. Low Temp. Phys. 1-2, 69-104 (1995). doi:10.1007/BF00753837

4. M. Loidl et al., Nucl. Instrum. Methods Phys. Res. A 465, 440-446 (2001). doi:10.1016/ S0168-9002(01)00621-0 